\title{
Generalized Courant-Snyder theory for coupled transverse dynamics of charged particles in electromagnetic focusing lattices
}

\author{
Hong Qin and Ronald C. Davidson \\ Plasma Physics Laboratory, Princeton University, Princeton, New Jersey 08543, USA
}

(Received 24 October 2008; published 25 June 2009)

\begin{abstract}
The Courant-Snyder theory gives a complete description of the uncoupled transverse dynamics of charged particles in electromagnetic focusing lattices. In this paper, the Courant-Snyder theory is generalized to the case of coupled transverse dynamics with two degrees of freedom. The generalized theory has the same structure as the original Courant-Snyder theory for one degree of freedom. The four basic components of the original Courant-Snyder theory, i.e., the envelope equation, phase advance, transfer matrix, and the Courant-Snyder invariant, all have their counterparts, with remarkably similar expressions, in the generalized theory presented here. In the generalized theory, the envelope function is generalized into an envelope matrix, and the envelope equation becomes a matrix envelope equation with matrix operations that are noncommutative. The generalized theory gives a new parametrization of the 4D symplectic transfer matrix that has the same structure as the parametrization of the $2 \mathrm{D}$ symplectic transfer matrix in the original Courant-Snyder theory. All of the parameters used in the generalized CourantSnyder theory correspond to physical quantities of importance, and this parametrization can provide a valuable framework for accelerator design and particle simulation studies. A time-dependent canonical transformation is used to develop the generalized Courant-Snyder theory. Applications of the new theory to strongly and weakly coupled dynamics are given. It is shown that the stability of coupled dynamics can be determined by the generalized phase advance developed. Two stability criteria are given, which recover the known results about sum and difference resonances in the weakly coupled limit.
\end{abstract}

DOI: 10.1103/PhysRevSTAB.12.064001

PACS numbers: 29.27.- a, 52.20.Dq

\section{INTRODUCTION AND THEORETICAL MODEL}

The transverse dynamics of a charged particle in a linear focusing lattice $\kappa_{q}(t)$ is described by an oscillator equation with time-dependent spring constant

$$
\ddot{q}+\kappa_{q}(t) q=0,
$$

where $q$ represents one of the transverse coordinates, either $x$ or $y$. For a quadrupole lattice, $\kappa_{x}(t)=-\kappa_{y}(t)$. The variable $t$ appearing in Eq. (1) is not really time, but rather the path length that plays the role of a timelike variable. The Courant-Snyder theory [1] gives a complete description of the solution to Eq. (1), and serves as the fundamen-

tal theory that underlies the design of modern accelerators and storage rings. There are four main components of the Courant-Snyder theory: the envelope equation, the phase advance, the transfer matrix, and the Courant-Snyder invariant. The Courant-Snyder theory can be summarized as follows. Because Eq. (1) is linear, the solution to Eq. (1) can be expressed as a time-dependent linear map from the initial conditions, i.e.,

$$
\left(\begin{array}{c}
q \\
\dot{q}
\end{array}\right)=M(t)\left(\begin{array}{c}
q_{0} \\
\dot{q}_{0}
\end{array}\right)
$$

$$
M(t)=\left(\begin{array}{cc}
\sqrt{\frac{\beta}{\beta_{0}}}\left[\cos \phi+\alpha_{0} \sin \phi\right] & \sqrt{\beta \beta_{0}} \sin \phi \\
-\frac{1+\alpha \alpha_{0}}{\sqrt{\beta \beta_{0}}} \sin \phi+\frac{\alpha_{0}-\alpha}{\sqrt{\beta \beta_{0}}} \cos \phi & \sqrt{\frac{\beta_{0}}{\beta}}[\cos \phi-\alpha \sin \phi]
\end{array}\right),
$$

where $q_{0}=q(t=0), \dot{q}_{0}=\dot{q}(t=0), \beta_{0}=\beta(t=0)$, and $\alpha_{0}=\alpha(t=0)$. The time-dependent functions $\alpha(t), \beta(t)$, and $\phi(t)$ in the transfer matrix $M(t)$ are directly related to the envelope function $w(t)$ by

$$
\begin{aligned}
& \beta(t)=w^{2}(t), \\
& \alpha(t)=-w \dot{w},
\end{aligned}
$$

$$
\phi(t)=\int_{0}^{t} \frac{d t}{\beta(t)} .
$$

The envelope function $w(t)$ satisfies the nonlinear envelope equation

$$
\ddot{w}+\kappa_{q}(t) w=w^{-3} .
$$

The physical meanings of $\beta^{-1}$ and $\phi$ correspond to the phase advance rate and the phase advance, respectively. 
The well-known Courant-Snyder invariant is

$$
I=\frac{q^{2}}{w^{2}}+(w \dot{q}-\dot{w} q)^{2} .
$$

The transfer matrix $M(t)$ is symplectic and has the following decomposition [2]:

$$
\begin{aligned}
M(t) & =\left(\begin{array}{cc}
\sqrt{\beta} & 0 \\
-\frac{\alpha}{\sqrt{\beta}} & \frac{1}{\sqrt{\beta}}
\end{array}\right)\left(\begin{array}{cc}
\cos \phi & \sin \phi \\
-\sin \phi & \cos \phi
\end{array}\right)\left(\begin{array}{cc}
\frac{1}{\sqrt{\beta_{0}}} & 0 \\
\frac{\alpha_{0}}{\sqrt{\beta_{0}}} & \sqrt{\beta_{0}}
\end{array}\right) \\
& =\left(\begin{array}{cc}
w & 0 \\
\dot{w} & \frac{1}{w}
\end{array}\right)\left(\begin{array}{cc}
\cos \phi & \sin \phi \\
-\sin \phi & \cos \phi
\end{array}\right)\left(\begin{array}{cc}
w_{0}^{-1} & 0 \\
-\dot{w}_{0} & w_{0}
\end{array}\right) .
\end{aligned}
$$

The Courant-Snyder theory was developed originally by Courant and Snyder for periodic systems with periodic $\kappa_{q}(t)$, using the method of the Floquet transformation. It is now realized that it is valid for general time-dependent systems, and it has been discovered (or rediscovered) many times during the past century in different disciplines [3-9]. A brief historical review can be found in Refs. [10,11]. When applying the Courant-Snyder theory to accelerators, the dynamics in the two transverse directions are considered to be decoupled. However, the coupling between the two transverse directions can be of considerable practical importance [12-14]. This effect was actually discussed by Courant and Snyder [1]. The general form of the Hamiltonian for the coupled transverse dynamics is given by

$$
H_{c}=\frac{1}{2} z A_{c} z^{T}
$$

where

$$
\begin{aligned}
& A_{c}=\left(\begin{array}{cc}
\kappa & R \\
R^{T} & I
\end{array}\right), \\
& z=\left(x, y, p_{x}, p_{y}\right), \\
& \kappa=\left(\begin{array}{cc}
\kappa_{x} & \kappa_{x y} \\
\kappa_{x y} & \kappa_{y}
\end{array}\right) .
\end{aligned}
$$

Here, the $2 \times 2$ matrix $\kappa(t)$ is time dependent and symmetric, $R$ is an arbitrary, time-dependent $2 \times 2$ matrix, and $I$ is the $2 \times 2$ unit matrix. The superscript $T$ denotes the transpose operation. The transverse dynamics are coupled through the $\kappa_{x y}(t)$ terms and the matrix $R$. A solenoidal lattice will induce nonvanishing $R$, and a skew quadrupole field will induce nonvanishing $\kappa_{x y}$. For a combined lattice with quadrupole, skew quadrupole, and solenoidal components,

$$
\begin{gathered}
\kappa=\left(\begin{array}{cc}
\Omega^{2}+\kappa_{q} & \kappa_{s q} \\
\kappa_{s q} & \Omega^{2}-\kappa_{q}
\end{array}\right), \\
R=\left(\begin{array}{cc}
0 & -\Omega \\
\Omega & 0
\end{array}\right),
\end{gathered}
$$

where $\kappa_{q}$ is the quadrupole focusing coefficient, $\Omega(t)=$ $e B_{z}(t) / 2 \gamma m c$ is one-half of the gyrofrequency associated with the solenoidal lattice, and $\kappa_{s q}$ is the skew quadrupole coefficient.

Since the dynamics is linear, the solution of the coupled system of equations obtained from $H_{c}$ is given by a transfer matrix $M_{c}(t)$, which is a time-dependent, $4 \times 4$ symplectic matrix [1]. Teng and Edwards [15-17] first systematically studied the transfer matrix $M_{c}(t)$, and derived a set of parametrizations for $M_{c}(t)$, which has been adopted in many lattice design and particle tracking codes, such as the MAD code [18]. Other possible parametrizations have also been considered [19-22]. However, these parametrizations lack connection to the original Courant-Snyder theory, which consists of several important physical quantities, such as the phase advance and the envelope dynamics.

In this paper, we extend the Courant-Snyder theory to the case of coupled transverse dynamics described by the Hamiltonian $H_{c}$ in Eq. (10) [23]. We show that the generalized Courant-Snyder theory gives a complete description of the coupled transverse dynamics, and has the same structure as the original Courant-Snyder theory for one degree of freedom. The four basic components of the original Courant-Snyder theory, i.e., the envelope equation, phase advance, transfer matrix, and the Courant-Snyder invariant, all have their counterparts, with remarkably similar expressions, in the generalized Courant-Snyder theory developed here. In the generalized theory, the envelope function $w$ is generalized to an envelope matrix, and the envelope equation becomes a matrix envelope equation with matrix operations that are not commutative. The generalized theory gives a parametrization of the 4D symplectic transfer matrix $M_{c}$ [Eqs. (77) and (108)] that has the same structure as the parametrization of the 2D symplectic transfer matrix $M$ [Eq. (9)] in the original Courant-Snyder theory. We will make use of a time-dependent linear transformation to develop the generalized Courant-Snyder theory. The fact that the linear transformation can be specified by a time-dependent symplectic matrix plays an important role in determining the structure of the generalized theory. This method was first proposed by Leach [24].

Because of the importance of the Courant-Snyder theory in accelerator physics, the generalization of the CourantSnyder theory has been undertaken in the past. An important generalization was constructed in 1992 by Dattoli et al. [25-27], who posed the question: "Can we adapt the coupled motion formalism to get a picture closer to the Courant-Snyder formulation?" [27]. Dattoli et al. generalized the Courant-Snyder theory by generalizing the standard Twiss parameters $\beta, \alpha$, and $\gamma$ into $2 \times 2$ matrixes. In this paper, we present a different solution to this fundamental question. We generalize the Courant-Snyder theory by generalizing the most fundamental elements of the Courant-Snyder theory, i.e., the envelope and the phase 
advance. The parameters of envelope and phase advance furnished by the Courant-Snyder theory are of vital importance for beam physics. These parameters describe the physical dimensions and the emittance of the beam, and set the foundation for many important concepts in beam physics, such as the Kapchinskij-Vladimirskij distribution function for intense beams with strong space-charge field. Therefore, the generalization scheme reported in the present paper makes a connection directly to the physics of the coupled dynamics. For example, using our generalization method, it is shown that the stability of coupled dynamics can be determined by the generalized phase advance. Two stability criteria are given, which recover the known results about sum and difference resonances in the weakly coupled limit. It is not surprising that a fundamental theory can be generalized by different methods from different angles, each of which may have its own advantages and range of applicability.

The present paper investigates the generalized CourantSnyder theory for coupled linear transverse dynamics. In a more general setting, it is necessary to parametrize the most comprehensive transverse map for the beam system with nonlinear effects in 6D phase space. Dragt has developed a normal form method to tackle this problem [28-34]. He showed that the one-turn map $\mathcal{M}$ for a ring can be symplectically normalized to a normal form $\mathcal{N}$ by a symplectic normalizing map $\mathcal{A}$, i.e. $\mathcal{N}=\mathcal{A} \mathcal{M} \mathcal{A}^{-1}$. This map $\mathcal{N}$ will be the same no matter what reference point is used. Its matrix part and nonlinear generators give full linear and nonlinear information about global ring properties including tunes, chromaticities to any order (in the static case), time-of-flight information to any order (in the static case), such as momentum compaction and phase slip factors, and anharmonicities to any order [32]. The matrix part and nonlinear generators of $\mathcal{A}$ give complete information about the lattice functions at the reference point. These lattice functions are periodic as one goes about the ring by choosing successive reference points. The matrix part of $\mathcal{A}$ provides the usual linear lattice functions, including dispersion, and the $\alpha, \beta, \gamma$ of Courant-Snyder theory, including their generalizations to six phase-space dimensions with arbitrary coupling. Dragt [32] pointed out that one advantage of the normal form methods is that it does not require numerical solutions of any linear and nonlinear differential equations. This was first done in the code MARYLIE [32]. These methods were subsequently incorporated into some versions of MAD. The code COSY INFINITY also incorporates several of these methods. Because the theoretical method in the present paper is developed only for the coupled linear transverse dynamics, we are able to achieve a generalized CourantSnyder theory having an exactly one-to-one correspondence with the original Courant-Snyder theory, which provides us with a specialized tool to investigate the detailed properties of the coupled transverse dynamics. For ex- ample, it enables us to study the stability of coupled dynamics from the generalized phase advance, and to generalize the well-known results about sum and difference resonances in the weakly coupled limit into two stability criteria for arbitrarily strong coupling.

This paper is organized as follows. In Sec. II, a method of time-dependent canonical transformation is described. In Sec. III, the generalized Courant-Snyder theory is developed. The concepts of envelope matrix, phase advance matrix, generalized transfer matrix, and the generalized Courant-Snyder invariant are introduced. In Sec. IV, we show that the generalized Courant-Snyder theory readily recovers, as special cases, the original Courant-Snyder theory for the case where the dynamics is uncoupled, and the well-known result for the coupled dynamics in a solenoidal lattice [35]. A numerical example of the generalized Courant-Snyder theory is given in Sec. $\mathrm{V}$ for the case of a focusing-off-defocusing-off (FODO) quadruple lattice with misalignment. The generalized Courant-Snyder theory is also applied to study the stability of the coupled transverse dynamics in Sec. V.

\section{TIME-DEPENDENT CANONICAL TRANSFORMATION}

We consider a linear, time-dependent Hamiltonian system with $n$ degree of freedom given by

$$
H=\frac{1}{2} z A(t) z^{T}, \quad z=\left(x_{1}, x_{2}, \ldots, x_{n}, p_{1}, p_{2}, \ldots, p_{n}\right) .
$$

Here, $A(t)$ is a $2 n \times 2 n$ time-dependent, symmetric matrix. The Hamiltonian in Eq. (10) has this form with $n=2$. We introduce a time-dependent linear canonical transformation [24]

$$
\bar{z}=S(t) z,
$$

such that in the new coordinate $\bar{z}$, the transformed Hamiltonian has the form

$$
\bar{H}=\frac{1}{2} \bar{z} \bar{A}(t) \bar{z}^{T},
$$

where $\bar{A}(t)$ is a targeted symmetric matrix. Because the transformation between $z$ and $\bar{z}$ is required to be a canonical transformation, we have

$$
\frac{\partial \bar{z}_{j}}{\partial z_{k}} J_{k l} \frac{\partial \bar{z}_{j}}{\partial z_{l}}=J_{i j}
$$

From Eq. (17), Eq. (19) is equivalent to

$$
S J S^{T}=J,
$$

i.e., the matrix $S$ specifying the coordination transformation between $z$ and $\bar{z}$ needs to be a symplectic matrix. In addition, the transformation $S(t)$ that renders this canonical transformation needs to satisfy another differential equation, which can be derived as follows. Hamilton's equation for $z$ is 


$$
\begin{gathered}
\dot{z}=J \nabla H, \\
J=\left(\begin{array}{cc}
0 & I \\
-I & 0
\end{array}\right),
\end{gathered}
$$

where $I$ is the $n \times n$ unit matrix and $J$ is the $2 n \times 2 n$ unit symplectic matrix of order $2 n$. Using index notation, we obtain

$$
\begin{aligned}
\dot{z}_{j} & =J_{i j} \frac{\partial H}{\partial z_{j}}=\frac{1}{2} J_{i j}\left(\delta_{l j} A_{l m} z_{m}+z_{l} A_{l k} \delta_{k j}\right) \\
& =\frac{1}{2} J_{i j}\left(A_{j m}+A_{m j}\right) z_{m}=J_{i j} A_{j m} z_{m} .
\end{aligned}
$$

Switching back to matrix notation without index, Eq. (22) can be expressed as

$$
\dot{z}=J A z \text {. }
$$

Since we require that in $\bar{z}$ the transformed Hamiltonian is given by Eq. (18), we also have

$$
\dot{\bar{z}}=J \bar{A} \bar{z} .
$$

Using the fact that $\bar{z}=S(t) z$, this is

$$
\dot{\bar{z}}=J \bar{A} \bar{z}=J \bar{A} S z \text {. }
$$

On the other hand, we can also calculate $\dot{\bar{z}}$ directly from Eq. (17), which is

$$
\dot{\bar{z}}=\dot{S} z+S \dot{z}=(\dot{S}+S J A) z
$$

Combining Eqs. (24) and (25) gives the differential equation

$$
\dot{S}=J \bar{A} S-S J A,
$$

which $S$ needs to satisfy if $\bar{z}=S(t) z$ is a canonical transformation.

This fact that the time-dependent symplectic matrix $S$ satisfying Eq. (26) gives the canonical transformation (17) between $H$ and $\bar{H}$ can also be proven constructively as the following. Suppose $A(t)$ and $\bar{A}(t)$ are any two (possibly) time-dependent symmetric matrices, and let $W$ be any desired symplectic matrix. We prove that there is a symplectic matrix $S(t)$ such that

$$
\dot{S}=J \bar{A} S-S J A
$$

and

$$
S\left(t_{0}\right)=W,
$$

where $t_{0}$ is any desired time. Define a matrix $U(t)$ by the differential equation

$$
\dot{U}=J A U
$$

with the initial condition $U\left(t_{0}\right)=I$. Then, by the usual arguments, $U$ will be symplectic. Similarly, define $\bar{U}(t)$ by the differential equation

$$
\dot{\bar{U}}=J \bar{A} \bar{U}
$$

with the initial condition $\bar{U}\left(t_{0}\right)=I$. Let $V(t)=U^{-1}(t)$, then

$$
\dot{V}=-V \dot{U} U^{-1}=-V J A U U^{-1}=-V J A .
$$

Define $S$ to be

$$
S(t)=\bar{U}(t) S\left(t_{0}\right) V(t)
$$

with $S\left(t_{0}\right)=W$. Direct calculation shows that

$$
\begin{aligned}
\dot{S} & =\bar{U} W V+\bar{U} W \dot{V}=J \bar{A} \bar{U} W V-\bar{U} W V J A \\
& =J \bar{A} S-S J A .
\end{aligned}
$$

Now suppose that $z(t)$ satisfies

$$
\dot{z}=J A z
$$

with the initial condition $z\left(t_{0}\right)=z_{0}$, then for $\bar{z}=S z$, we have

$$
\dot{\bar{z}}=\dot{S} z+S \dot{z}=[J \bar{A} S-S J A] z+S J A=J \bar{A} S z=J \bar{A} \bar{z} .
$$

The initial condition for $\bar{z}$ can be chosen to be

$$
\bar{z}\left(t_{0}\right)=S\left(t_{0}\right) z\left(t_{0}\right)=W z_{0} .
$$

If we specify $\bar{z}\left(t_{0}\right)$ and $z\left(t_{0}\right)$ instead, then we can always find a symplectic matrix $W$ as the initial condition for $S$ satisfying Eq. (36), because the symplectic group acts transitively on phase space.

Using the transformation given by $S$, we can transform the Hamiltonian given by $A(t)$ into any Hamiltonian specified by $\bar{A}(t)$. In particular, if we select the target matrix $\bar{A}=$ 0 , then the Hamiltonian system given by $A(t)$ is solved by the transformation which transforms $H=z A z^{T}$ into $\bar{H}=$ $\bar{z} \bar{A} \bar{z}^{T}=0$.

\section{GENERALIZED COURANT-SNYDER THEORY FOR COUPLED TRANSVERSE DYNAMICS}

We are now ready to develop the generalized CourantSnyder theory for coupled transverse dynamics described by the Hamiltonian $H_{c}$ in Eq. (10), using the technique of time-dependent canonical transformation developed in Sec. II. Our objective is to solve the coupled system by finding the transfer matrix between the initial condition $z_{0}=\left(x_{0}, y_{0}, p_{x 0}, p_{y 0}\right)$ and $z=\left(x, y, p_{x}, p_{y}\right)$ at time $t$. We accomplish this goal by a series of time-dependent canonical transformations. We start from the Hamiltonian given by

$$
\begin{gathered}
H_{c 0}=\frac{1}{2} z A_{c 0} z^{T}, \\
A_{c 0}=\left(\begin{array}{cc}
\kappa & 0 \\
0 & I
\end{array}\right), \\
\kappa=\left(\begin{array}{cc}
\kappa_{x} & \kappa_{x y} \\
\kappa_{x y} & \kappa_{y}
\end{array}\right) .
\end{gathered}
$$


The Hamiltonian $H_{c 0}$ is the special case of $H_{c}$ in Eq. (10) with $\Omega=0$, and corresponds to the case of coupling induced by factors other than solenoidal field, for example, by a skew quadrupole field. We first develop the generalized Courant-Snyder theory for $H_{c 0}$ and determine the transfer matrix $M_{c 0}(t)$. After this, we will determine the transformation which transforms general $H_{c}$ into $H_{c 0}$, and generate the transfer matrix $M_{c}(t)$ for $H_{c}$.

\section{A. Generalized Courant-Snyder theory for $\boldsymbol{H}_{\boldsymbol{c} 0}$}

There are two steps to solve the system given by $H_{c 0}$. The first step is to transform $H_{c 0}$ into

$$
\begin{gathered}
\bar{H}_{c 0}=\frac{1}{2} \bar{z} \bar{A}_{c 0} \bar{z}^{T}, \\
\bar{A}_{c 0}=\left(\begin{array}{cc}
\beta_{I} & 0 \\
0 & \beta_{I}
\end{array}\right),
\end{gathered}
$$

and the second step is to transform $\bar{H}_{c 0}$ into $\overline{\bar{H}}_{c 0}=0$. Here, $\beta_{I}$ is a time-dependent $2 \times 2$ matrix to be determined. We will demonstrate shortly that $\beta_{I}$ is the inverse of the generalized $\beta$ function matrix, as suggested by its notation. The physics that appears in the first step is the envelope matrix and the noncommutative matrix envelope equation. The physics that appears in the second step is the phase advance. Let $\bar{z}=S z$ be the transformation that transforms $H_{c 0}$ into $\bar{H}_{c 0}$. From Eq. (26), the differential equation for $S$ is

$$
\dot{S}=J \bar{A}_{c 0} S-S J A_{c 0} .
$$

To cast it into the $2 \times 2$ block form, let

$$
S=\left(\begin{array}{ll}
S_{1} & S_{2} \\
S_{3} & S_{4}
\end{array}\right)
$$

where $S_{1}, S_{2}, S_{3}$, and $S_{4}$ are $2 \times 2$ matrixes. Equation (42) can then be expressed as

$$
\begin{aligned}
\left(\begin{array}{ll}
\dot{S}_{1} & \dot{S}_{2} \\
\dot{S}_{3} & \dot{S}_{4}
\end{array}\right)= & \left(\begin{array}{cc}
0 & I \\
-I & 0
\end{array}\right)\left(\begin{array}{cc}
\beta_{I} & 0 \\
0 & \beta_{I}
\end{array}\right)\left(\begin{array}{ll}
S_{1} & S_{2} \\
S_{3} & S_{4}
\end{array}\right) \\
& -\left(\begin{array}{ll}
S_{1} & S_{2} \\
S_{3} & S_{4}
\end{array}\right)\left(\begin{array}{cc}
0 & I \\
-I & 0
\end{array}\right)\left(\begin{array}{ll}
\kappa & 0 \\
0 & I
\end{array}\right),
\end{aligned}
$$

which is equivalent to

$$
\begin{gathered}
\dot{S}_{1}=\beta_{I} S_{3}+S_{2} \kappa, \\
\dot{S}_{2}=\beta_{I} S_{4}-S_{1}, \\
\dot{S}_{3}=-\beta_{I} S_{1}+S_{4} \kappa, \\
\dot{S}_{4}=-\beta_{I} S_{2}-S_{3} .
\end{gathered}
$$

Because $\beta_{I}$ is still undetermined, we let $S_{2}=0$, and obtain

$$
\dot{S}_{1}=\beta_{I} S_{3},
$$

$$
\begin{gathered}
S_{1}=\beta_{I} S_{4}, \\
\dot{S}_{3}=-\beta_{I} S_{1}+S_{4} \kappa, \\
\dot{S}_{4}=-S_{3} .
\end{gathered}
$$

Setting $S_{2}=0$ in general does not violate the symplectic condition. It can be shown that there are symplectic matrices with $S_{2}=0$. Equations (49), (50), and (52) give

$$
\dot{\beta}_{I} S_{4}+2 \beta_{I} \dot{S}_{4}=0 .
$$

Furthermore, Eqs. (49), (51), and (52) give

$$
\ddot{S}_{4}=\beta_{I}^{2} S_{4}-S_{4} \kappa \text {. }
$$

Now define $\beta \equiv \beta_{I}^{-1}$ and $w \equiv S_{4}$. Here, we rename $S_{4}$ to be $w$, because it will be clear shortly that $S_{4}$ corresponds to the envelope matrix, and it is conventional to denote the envelope function by $w$. In terms of $\beta$ and $w$, Eq. (54) can be expressed as

$$
\ddot{w}=\beta^{-2} w-w \kappa .
$$

We now invoke the fact that $S$ can always be chosen to be symplectic with

$$
S_{1} S_{4}^{T}-S_{2} S_{3}^{T}=I
$$

which gives

$$
\beta^{-1} w w^{T}=I,
$$

or

$$
\beta=w w^{T} .
$$

Combining Eqs. (55) and (58), we obtain the envelope equation for the $2 \times 2$ envelope matrix $w$, i.e.,

$$
\ddot{w}+w \kappa=\left(w^{-1}\right)^{T} w^{-1}\left(w^{-1}\right)^{T} .
$$

The similarity between the matrix envelope equation (59) and the original envelope equation (7) is remarkable. The unique feature of Eq. (59) is that it is noncommutative. This is because Eq. (59) is written in terms of matrix functions, and matrix operations are noncommutative in general. For example, the second term in Eq. (59) cannot be written as $\kappa w$. It can be shown that Eq. (59) recovers Eq. (7) as a special case, which will be discussed in Sec. IV. The transformation for this step is

$$
\begin{gathered}
\bar{z}=S z, \\
S=\left(\begin{array}{cc}
\left(w^{-1}\right)^{T} & 0 \\
-\dot{w} & w
\end{array}\right),
\end{gathered}
$$

and the inverse transformation is

$$
z=S^{-1} \bar{z}
$$




$$
S^{-1}=\left(\begin{array}{cc}
w^{T} & 0 \\
w^{-1} \dot{w} w^{T} & w^{-1}
\end{array}\right) .
$$

The matrix $S^{-1}$ is the noncommutative generalization of the first matrix in the expression of the transfer matrix $M$ for the original Courant-Snyder theory, i.e., the first term on the right-hand side of Eq. (9). From Eq. (58), note that $\beta^{-1}$ is symmetric, which ensures that $\bar{A}_{c 0}$ is symmetric.

The next step is to transform $\bar{H}_{c 0}$ into $\overline{\bar{H}}_{c 0}=0$ with $\overline{\bar{A}}_{c 0}=0$ by a transformation specified by $P$ :

$$
\overline{\bar{z}}=P \bar{z} \text {. }
$$

Following the same procedure described in Sec. II, the differential equation for $P$ is

$$
\begin{gathered}
\dot{P}_{1}=P_{2} \beta^{-1}, \\
\dot{P}_{2}=-P_{1} \beta^{-1}, \\
\dot{P}_{3}=P_{4} \beta^{-1}, \\
\dot{P}_{4}=-P_{3} \beta^{-1} .
\end{gathered}
$$

Equations (65) and (66) are decoupled from Eqs. (67) and (68), and Eqs. (65)-(68) admit solutions with

$$
\begin{gathered}
P_{4}=P_{1}, \\
P_{3}=-P_{2} .
\end{gathered}
$$

The transformation matrix is

$$
P=\left(\begin{array}{cc}
P_{1} & P_{2} \\
-P_{2} & P_{1}
\end{array}\right)
$$

where $P_{1}$ and $P_{2}$ are determined from Eqs. (65) and (66). From the fact that $P$ belongs to $S p(4, R)$, we can readily show that

$$
P P^{T}=I, \quad \text { and } \quad \operatorname{Det}(P)=1 .
$$

Therefore, $P$ corresponds to a rotation in the $4 \mathrm{D}$ phase space, $P \in S O(4)$. In this sense, $P^{T}$ is the $4 \mathrm{D}$ noncommutative generalization of the $2 \mathrm{D}$ rotation matrix in the expression of the transfer matrix $M$ for the original CourantSnyder theory, i.e., the second term on the right-hand side of Eq. (9). The differential equation for $P$ can be written as

$$
\begin{gathered}
\dot{P}=P \dot{\phi}, \\
\dot{\phi} \equiv\left(\begin{array}{cc}
0 & -\beta^{-1} \\
\beta^{-1} & 0
\end{array}\right)=\left(\begin{array}{cc}
0 & -\left(w^{-1}\right)^{T} w^{-1} \\
\left(w^{-1}\right)^{T} w^{-1} & 0
\end{array}\right) .
\end{gathered}
$$

Because $\dot{\phi}^{T}=-\dot{\phi}$, it follows that $\dot{\phi}$ belongs to the Lie algebra $s o(4)$, i.e., $\dot{\phi}$ is an infinitesimal generator of a $4 \mathrm{D}$ rotation. In another word, $\dot{\phi}$ is an "angular velocity" in $4 \mathrm{D}$ space, which is equivalent to a phase advance rate in $4 \mathrm{D}$ space. The 4D phase advance rate $\dot{\phi}$ is determined from the $2 \times 2$ matrix $\beta^{-1}=\left(w^{-1}\right)^{T} w^{-1}$, which is remarkably similar to the phase advance rate $\beta^{-1}=1 / w^{2}$ in the original Courant-Snyder theory for one degree of freedom [see Eqs. (4) and (6)]. In Sec. IV, we will show how $P$ and $\phi$ recover the 2D rotation and phase advance for the special case of uncoupled dynamics.

Because $\overline{\bar{H}}_{c 0}=0$, the dynamics of $\overline{\bar{z}}$ is trivial, i.e., $\overline{\bar{z}}=$ $\overline{\bar{z}}_{0}$, and we have solved the Hamiltonian system $H_{c 0}$ in $\overline{\bar{z}}$. From $\overline{\bar{z}}=P S z$ and $\overline{\bar{z}}=\overline{\bar{z}}_{0}$, we obtain the linear map between $z_{0}$ and $z$, i.e.,

$$
z=S^{-1} P^{-1} \overline{\bar{z}}=S^{-1} P^{-1} \overline{\bar{z}}_{0}=S^{-1} P^{-1} P_{0} S_{0} z_{0} .
$$

Because $P \in S O(4, R)$, without loss of generality we select the initial condition $P_{0}=P(t=0)=I$, to obtain

$$
\begin{aligned}
z=M_{c 0} z_{0}, & \\
M_{c 0} & =S^{-1} P^{-1} S_{0} \\
& =\left(\begin{array}{cc}
w^{T} & 0 \\
w^{-1} \dot{w} w^{T} & w^{-1}
\end{array}\right)\left(\begin{array}{cc}
P_{1} & -P_{2} \\
P_{2} & P_{1}
\end{array}\right)\left(\begin{array}{cc}
\left(w_{0}^{-1}\right)^{T} & 0 \\
-\dot{w}_{0} & w_{0}
\end{array}\right) .
\end{aligned}
$$

The transfer matrix $M_{c 0}$ in Eq. (77) is the 4D noncommutative generalization of the transfer matrix in Eq. (9) for one degree of freedom. The similarities between $M_{c 0}$ and $M$ is evident from Eqs. (77) and (9).

We note that Eq. (77) has the general format of Eq. (38) of Ref. [28], which is valid for any general linear or nonlinear lattice. The specific expressions of the matrix elements in Eq. (77) are of course not true for an arbitrary linear or nonlinear lattice. They are only correct for the coupled linear lattice under investigation here.

Because $\overline{\bar{z}}=P S z$ is a constant $1 \times 2$ vector, any function of $\overline{\bar{z}}=P S z$ is also an invariant. Consequently, for every constant $2 \times 2$ matrix $C$,

$$
I_{C}=\overline{\bar{z}}^{T} C \overline{\bar{z}}
$$

is an invariant of the dynamics. The original CourantSnyder (CS) invariant in Eq. (8) for one degree of freedom is an invariant independent of the phase advance. The generalized Courant-Snyder invariant for 4D coupled dynamics corresponding to the original Courant-Snyder invariant is

$$
I_{\mathrm{CS}}=\overline{\bar{z}}^{T} \overline{\bar{z}}=z^{T} S^{T} P^{T} P S z=z^{T} S^{T} S z,
$$

where the phase advance has been removed due to the fact that $P$ is a 4D rotation. Equation (79) is a special case of Eq. (78) for $C=I$. For a ring, we can also construct invariants of dynamics using the one-turn map [1]

$$
M_{c T}(t)=\left(\begin{array}{cc}
w^{T} & 0 \\
w^{-1} \dot{w} w^{T} & w^{-1}
\end{array}\right) P_{T}\left(\begin{array}{cc}
w^{-1 T} & 0 \\
-\dot{w} & w
\end{array}\right),
$$

where $P_{T}(t)$ is the generalized one-turn phase advance matrix. For every integer $k$, there is an invariant 


$$
I_{k} \equiv z^{T} U_{k} z=\text { const }
$$

where $U_{k}$ is given by

$$
U_{k}(t)=J M_{c T}^{k}-M_{c T}^{T k} J
$$

If we use the normal form method [28-32] to decouple the two transverse dynamics, each freedom will have its own Courant-Snyder invariant. Of course, only two invariants are functionally independent, and all other invariants can be expressed in terms of these two functionally independent invariants.

\section{B. Generalized Courant-Snyder theory for $\boldsymbol{H}_{\boldsymbol{c}}$}

Our strategy to solve for the $H_{c}$ system is to transform $H_{c}$ into the form of $H_{c 0}$, using the same transformation technique. Let

$$
\tilde{z}=Q z
$$

such that $H_{c}=z A_{c} z^{T}$ is transformed into

$$
\begin{aligned}
& H_{c 0}=\frac{1}{2} \tilde{z} \tilde{A}_{c 0} \tilde{z}^{T}, \\
& \tilde{A}_{c 0}=\left(\begin{array}{ll}
\tilde{\kappa} & 0 \\
0 & I
\end{array}\right),
\end{aligned}
$$

where $\tilde{\kappa}$ is to be determined from $A_{c}$. The differential equation for $Q$ is

$$
\begin{aligned}
\left(\begin{array}{cc}
\dot{Q}_{1} & \dot{Q}_{2} \\
\dot{Q}_{3} & \dot{Q}_{4}
\end{array}\right)= & \left(\begin{array}{cc}
0 & I \\
-I & 0
\end{array}\right)\left(\begin{array}{cc}
\tilde{\kappa} & 0 \\
0 & I
\end{array}\right)\left(\begin{array}{ll}
Q_{1} & Q_{2} \\
Q_{3} & Q_{4}
\end{array}\right) \\
& -\left(\begin{array}{ll}
Q_{1} & Q_{2} \\
Q_{3} & Q_{4}
\end{array}\right)\left(\begin{array}{cc}
0 & I \\
-I & 0
\end{array}\right)\left(\begin{array}{cc}
\kappa & R \\
R^{T} & I
\end{array}\right)
\end{aligned}
$$

In element format, Eq. (86) can be expressed as

$$
\begin{gathered}
\dot{Q}_{1}=Q_{3}+Q_{2} \kappa-Q_{1} R^{T}, \\
\dot{Q}_{2}=Q_{4}+Q_{2} R-Q_{1}, \\
\dot{Q}_{3}=-\tilde{\kappa} Q_{1}+Q_{4} \kappa-Q_{3} R^{T}, \\
\dot{Q}_{4}=-\tilde{\kappa} Q_{2}+Q_{4} R-Q_{3} .
\end{gathered}
$$

Since $\tilde{\kappa}$ is not specified, we can set $Q_{2}=0$ and the system reduces to

$$
\begin{gathered}
Q_{1}=Q_{4} \\
\dot{Q}_{4}=Q_{4} R-Q_{3}, \\
\dot{Q}_{4}=Q_{3}-Q_{4} R^{T}, \\
\dot{Q}_{3}=-\tilde{\kappa} Q_{4}+Q_{4} \kappa-Q_{3} R^{T},
\end{gathered}
$$

which leads to

$$
\begin{gathered}
Q_{3}=\frac{Q_{4}}{2}\left(R+R^{T}\right), \\
\dot{Q}_{4}=\frac{Q_{4}}{2}\left(R-R^{T}\right), \\
\tilde{\kappa}=Q_{4}\left[\kappa-\frac{\left(R+R^{T}\right) R^{T}}{2}-\frac{\left(R-R^{T}\right)\left(R+R^{T}\right)}{4}\right. \\
\left.-\frac{\dot{R}+\dot{R}^{T}}{2}\right] Q_{4}^{-1} .
\end{gathered}
$$

The transformation matrix is

$$
Q=\left(\begin{array}{cc}
Q_{4} & 0 \\
\frac{Q_{4}\left(R+R^{T}\right)}{2} & Q_{4}
\end{array}\right)
$$

As before, $Q$ is symplectic, which implies $Q_{4} Q_{4}^{T}=I$, and $Q_{4}$ is a $2 \mathrm{D}$ rotation, i.e., $Q_{4} \in O(2)$. These properties are valid for any $2 \times 2$ matrix $R$.

For $R$ in Eq. (15) corresponding to solenoidal lattice, $R+R^{T}=0$, and the transformation is further simplified to become

$$
\begin{gathered}
\tilde{\kappa}=Q_{4} \kappa Q_{4}^{-1} \\
Q=\left(\begin{array}{cc}
Q_{4} & 0 \\
0 & Q_{4}
\end{array}\right) \\
\dot{Q}_{4}=Q_{4} R=\Omega Q_{4}\left(\begin{array}{cc}
0 & -1 \\
1 & 0
\end{array}\right) .
\end{gathered}
$$

Equation (101) has the following solution:

$$
\begin{gathered}
Q_{4}=\left(\begin{array}{cc}
\cos \psi & -\sin \psi \\
\sin \psi & \cos \psi
\end{array}\right), \\
\dot{\psi}=\Omega,
\end{gathered}
$$

which can be easily verified by direct substitution. Obviously, $Q_{4}$ is a $2 \mathrm{D}$ rotation with angular velocity at one-half of the gyrofrequency. The transformation $Q$ is a $4 \mathrm{D}$ rotation with

$$
Q^{-1}=Q^{T}=\left(\begin{array}{cc}
Q_{4}^{T} & 0 \\
0 & Q_{4}^{T}
\end{array}\right)=\left(\begin{array}{cc}
Q_{4}^{-1} & 0 \\
0 & Q_{4}^{-1}
\end{array}\right) .
$$

The 4D rotation represented by $Q$ is a rotation of the same amount in both the $\left(x, p_{x}\right)$ plane and the $\left(y, p_{y}\right)$ plane. Such rotations in 4D geometry are called isocline double rotations [36].

We can now use the procedure developed in Sec. III A to transform $H_{c 0}$ into $\overline{\bar{H}}_{c 0}=0$. Overall, the transformation from $H_{c}$ into $\overline{\bar{H}}_{c 0}=0$ is

$$
\overline{\bar{z}}=P S Q z
$$

and the dynamics of $\overline{\bar{z}}$ is trivial with $\overline{\bar{z}}=\overline{\bar{z}}_{0}$. The linear mapping between $z_{0}$ and $z$ is 


$$
\begin{aligned}
z & =Q^{-1} S^{-1} P^{-1} \overline{\bar{z}}=Q^{-1} S^{-1} P^{-1} \overline{\bar{z}}_{0} \\
& =Q^{-1} S^{-1} P^{-1} P_{0} S_{0} Q_{0} z_{0} .
\end{aligned}
$$

Both $P$ and $Q$ are 4D rotations, and we can chose the initial conditions such that $P_{0}=I$ and $Q_{0}=I$.

Finally, we assemble all the calculations together into the following final format. For the coupled transverse dynamics given by the Hamiltonian $H_{c}$ in Eqs. (10), (14), and (15), we express

$$
z(t)=M_{c} z_{0},
$$

where $M_{c}$, the transfer matrix between the initial condition $z_{0}$ and $z(t)$, is given by

$$
\begin{gathered}
M_{c}=Q^{-1} S^{-1} P^{-1} S_{0}, \\
Q^{-1}=\left(\begin{array}{cc}
Q_{4}^{T} & 0 \\
0 & Q_{4}^{T}
\end{array}\right) \in S O(4), \\
Q_{4}=\left(\begin{array}{cc}
\cos \psi & -\sin \psi \\
\sin \psi & \cos \psi
\end{array}\right) \in S O(2), \\
\dot{\psi}=\Omega, \\
S^{-1}=\left(\begin{array}{cc}
w^{T} & 0 \\
w^{-1} \dot{w} w^{T} & w^{-1}
\end{array}\right), \\
S_{0}=\left(\begin{array}{cc}
\left(w_{0}^{-1}\right)^{T} & 0 \\
-\dot{w}_{0} & w_{0}
\end{array}\right), \\
P^{-1}=\left(\begin{array}{cc}
P_{1} & -P_{2} \\
P_{2} & P_{1}
\end{array}\right) \in S O(4) .
\end{gathered}
$$

Here, $w$ is the $2 \times 2$ envelope matrix satisfying the following noncommutative matrix envelope equation:

$$
\ddot{w}+w \tilde{\kappa}=\left(w^{-1}\right)^{T} w^{-1}\left(w^{-1}\right)^{T},
$$

and $\left(w_{0}, \dot{w}_{0}\right)$ denotes the initial conditions for $w$. The rotation matrix $P^{-1}$ is determined from the generalized phase advance equations

$$
\begin{gathered}
\dot{P}_{1}=P_{2} \beta^{-1}, \\
\dot{P}_{2}=-P_{1} \beta^{-1},
\end{gathered}
$$

where $\beta^{-1}=\left(w^{-1}\right)^{T} w^{-1}$ is the matrix phase advance rate determined from the envelope matrix $w$. Any function of $\overline{\bar{z}}=P S Q z$ is an invariant of the dynamics. The generalized Courant-Snyder invariant is

$$
I_{c}^{\mathrm{CS}}=z^{T} Q^{T} S^{T} S Q z,
$$

where the phase advance in the 4D rotation $P$ is canceled by the counter-rotation $P^{T}$.

These results are the noncommutative generalization of the Courant-Snyder theory for one degree of freedom to the coupled transverse dynamics of 2 degrees of freedom.

\section{SPECIAL CASES}

In this section we show that the generalized CourantSnyder theory developed for coupled transverse dynamics recovers the original Courant-Snyder theory for dynamics with one degree of freedom, and the well-known transfer matrix for a solenoidal focusing lattice, as special cases.

First, we investigate the case of uncoupled transverse dynamics given by $H_{c 0}$ with $\kappa_{x y}=0$. Because $\kappa$ is diagonal, the matrix envelope equation (59) admits solutions with diagonal envelope matrix

$$
w=\left(\begin{array}{cc}
w_{x} & 0 \\
0 & w_{y}
\end{array}\right) .
$$

Consequently, every matrix in Eq. (59) is diagonal, and the matrix operation is commutative. The matrix envelope equation reduces to two decoupled envelope equations of the conventional form for $w_{x}$ and $w_{y}$, i.e.,

$$
\begin{gathered}
\ddot{w}_{x}+w_{x} \kappa_{x}=w_{x}^{-3}, \\
\ddot{w}_{y}+w_{y} \kappa_{y}=w_{y}^{-3} .
\end{gathered}
$$

The $2 \times 2$ matrix of phase advance rate $\beta^{-1}$ reduces to a diagonal matrix as well, i.e.,

$$
\beta^{-1}=\left(\begin{array}{cc}
w_{x}^{-2} & 0 \\
0 & w_{y}^{-2}
\end{array}\right)
$$

The differential equation for the 4D rotation $P$ is simplified to become

$$
\begin{gathered}
\dot{P}_{1}=P_{2}\left(\begin{array}{cc}
w_{x}^{-2} & 0 \\
0 & w_{y}^{-2}
\end{array}\right), \\
\dot{P}_{2}=-P_{1}\left(\begin{array}{cc}
w_{x}^{-2} & 0 \\
0 & w_{y}^{-2}
\end{array}\right),
\end{gathered}
$$

which admits a diagonal solution

$$
\begin{aligned}
& P_{1}=\left(\begin{array}{cc}
P_{1 x} & 0 \\
0 & P_{1 y}
\end{array}\right), \\
& P_{2}=\left(\begin{array}{cc}
P_{2 x} & 0 \\
0 & P_{2 y}
\end{array}\right) .
\end{aligned}
$$

Note that the $\left(P_{1 x}, P_{2 x}\right)$ component is decoupled from the $\left(P_{1 y}, P_{2 y}\right)$ component, and they satisfy two sets of equations independently, i.e.,

$$
\begin{gathered}
\dot{P}_{1 x}=P_{2 x} w_{x}^{-2}, \\
\dot{P}_{2 x}=-P_{1 x} w_{x}^{-2},
\end{gathered}
$$

and

$$
\dot{P}_{1 y}=P_{2 y} w_{y}^{-2},
$$




$$
\dot{P}_{2 y}=-P_{1 y} w_{y}^{-2} .
$$

The solutions are

$$
\begin{gathered}
P_{1 x}=\cos \phi_{x}, \\
P_{2 x}=-\sin \phi_{x}, \\
\dot{\phi}_{x}=\frac{1}{w_{x}^{2}},
\end{gathered}
$$

and

$$
\begin{gathered}
P_{1 y}=\cos \phi_{y}, \\
P_{2 y}=-\sin \phi_{y}, \\
\dot{\phi}_{y}=\frac{1}{w_{y}^{2}} .
\end{gathered}
$$

Now, the transfer matrix can be expressed as

$$
\begin{aligned}
M= & \left(\begin{array}{cccc}
w_{x} & 0 & 0 & 0 \\
0 & w_{y} & 0 & 0 \\
\dot{w}_{x} & 0 & w_{x}^{-1} & 0 \\
0 & \dot{w}_{y} & 0 & w_{y}^{-1}
\end{array}\right) \\
& \times\left(\begin{array}{cccc}
\cos \phi_{x} & 0 & -\sin \phi_{x} & 0 \\
0 & \cos \phi_{y} & 0 & -\sin \phi_{y} \\
\sin \phi_{x} & 0 & \cos \phi_{x} & 0 \\
0 & \sin \phi_{y} & 0 & \cos \phi_{y}
\end{array}\right) \\
& \times\left(\begin{array}{cccc}
w_{x 0}^{-1} & 0 & 0 & 0 \\
0 & w_{y 0}^{-1} & 0 & 0 \\
-\dot{w}_{x 0} & 0 & w_{x 0} & 0 \\
0 & -\dot{w}_{y 0} & 0 & w_{y 0}
\end{array}\right) .
\end{aligned}
$$

Apparently, the $\left(x, p_{x}\right)$ dynamics and the $\left(y, p_{y}\right)$ dynamics are decoupled, and the transfer matrices for $\left(x, p_{x}\right)$ and $\left(y, p_{y}\right)$ extracted from Eq. (137) are identical to that in Eq. (9) for one degree of freedom. In summary, for uncoupled transverse dynamics, the general theory becomes commutative, and the theory reduces to the original Courant-Snyder theory independently for each transverse direction.

The second special case we investigate corresponds to the case of coupled transverse dynamics in a solenoidal lattice. Note that the solenoidal lattice need not be periodic. This is the special case where

$$
\begin{aligned}
& \kappa=\left(\begin{array}{cc}
\Omega^{2} & 0 \\
0 & \Omega^{2}
\end{array}\right), \\
& R=\left(\begin{array}{cc}
0 & -\Omega \\
\Omega & 0
\end{array}\right) .
\end{aligned}
$$

The symplectic canonical transformation given by $Q$ in Eq. (100) shows that the isocline double rotation of the 4D phase space in the $\left(x, p_{x}\right)$ and $\left(y, p_{y}\right)$ planes with instantaneous angular velocity $\dot{\psi}=\Omega$ transforms the coupled dynamics into two decoupled transverse dynamical equations with time-dependent focusing matrix

$$
\tilde{\kappa}=Q_{4}\left(\begin{array}{cc}
\Omega^{2} & 0 \\
0 & \Omega^{2}
\end{array}\right) Q_{4}^{-1}=\left(\begin{array}{cc}
\Omega^{2} & 0 \\
0 & \Omega^{2}
\end{array}\right) .
$$

In the rotating frame, the two transverse dynamical equations are decoupled, and each is described by the original Courant-Snyder theory. This is of course the well-known result for a solenoidal lattice [35].

\section{APPLICATIONS}

In this section, we present two applications of the generalized Courant-Snyder theory developed in previous sections. The first application is the numerical calculation of the transfer matrix for a periodic quadrupole FODO lattice with the middle magnet being misaligned by a small angle $\theta$. We consider a $10 \mathrm{MeV} \mathrm{Li}^{+}$beam with $A=7$, typical of the neutralized drift compression experiment II that is being constructed $[37,38]$. The lattice length is chosen to be $L=2 \mathrm{~m}$, magnetic gradient $B_{q}^{\prime}=1.172 \mathrm{~T} / \mathrm{m}$, and the filling factor $\eta=0.15$. The misaligned magnet induces a skew quadrupole component of the form [13]

$$
\kappa_{x y}=\kappa_{y x}=\kappa_{q} \sin 2 \theta .
$$

The strength of the quadrupole component of the misaligned magnet is reduced to

$$
\kappa_{x}=-\kappa_{y}=\kappa_{q} \cos 2 \theta .
$$

For comparison, the $\kappa_{x}$ and $\kappa_{x y}$ coefficients of the FODO lattice are plotted in Fig. 1 for the cases without misalignment $(\theta=0)$ and with misalignment of $\theta=5.7^{\circ}$. The calculation for the coupled dynamics is carried out in dimensionless parameters. The length is normalized by the lattice period $L$, and the velocity is normalized by the beam velocity $V_{b}$. The normalized strength of the quadrupole focusing field is $k_{q} \equiv e_{b} B_{q}^{\prime} / \gamma_{b} m_{b} \beta_{b} c^{2}=15$ [39]. The envelope matrix equation (59) is numerically solved for matched solutions using the standard shooting method. The results are displayed in Fig. 2 for $\theta=0$ and $\theta=5.7^{\circ}$, where $w_{1}, w_{2}, w_{3}$, and $w_{4}$ are the four elements of $w$, i.e.,

$$
w=\left(\begin{array}{ll}
w_{1} & w_{2} \\
w_{3} & w_{4}
\end{array}\right) .
$$

The numerical results show that for the case without misalignment $(\theta=0)$, the envelope matrix $w$ is diagonal, as expected from the discussion in Sec. IV. The $w_{1}$ and $w_{4}$ functions for $\theta=0$ are the familiar $x$ and $y$ envelopes for the uncoupled case. For $\theta=5.7^{\circ}$, it is evident that the envelope matrix becomes nondiagonal due to the coupling between the two transverse degree of freedoms induced by 

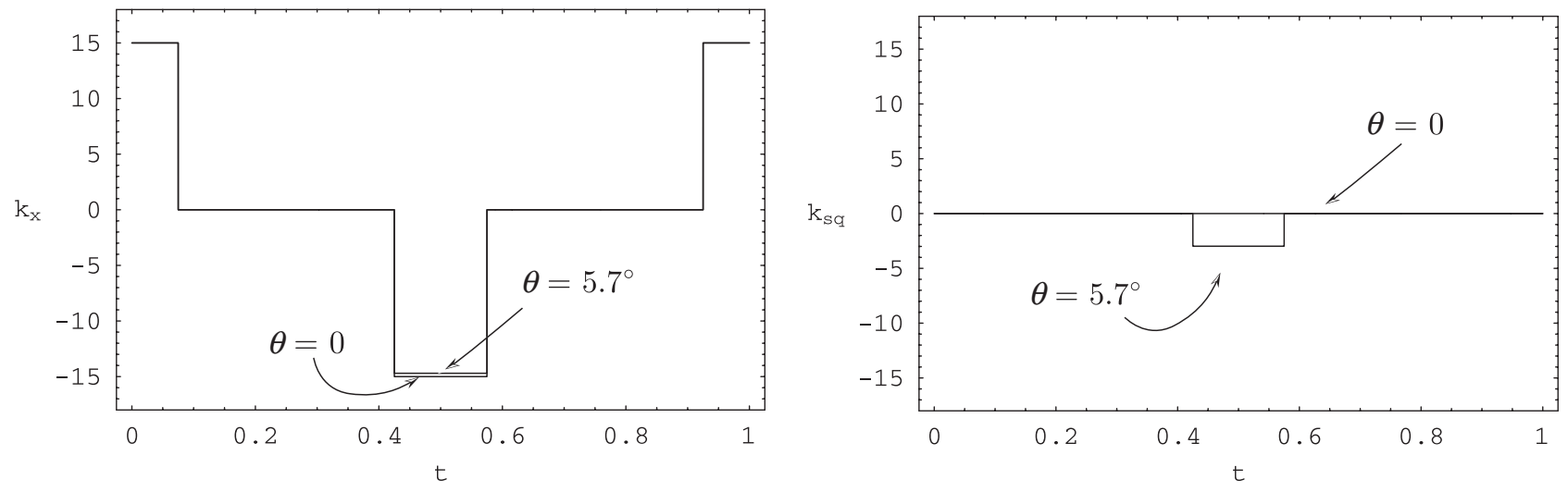

FIG. 1. The $\kappa_{x}$ and $\kappa_{x y}$ coefficients of the FODO lattice for the cases without misalignment $(\theta=0)$ and with a misalignment of $\theta=5.7^{\circ}$ for the middle magnet. The $t$ coordinate is normalized by the periodicity of the lattice. The misalignment introduces a skew quadrupole component, and reduces the strength of the quadrupole component of the focusing field.

the skew quadrupole component. The amplitude of the nondiagonal elements $w_{2}$ and $w_{3}$ are relatively small because the misalignment is weak. The elements of the matrix $P$ are determined by solving Eqs. (65) and (66), and the results are plotted in Fig. 3, where $P_{1 x}, P_{1 x y}, P_{1 y x}$, $P_{1 y}, P_{2 x}, P_{2 x y}, P_{2 y x}$, and $P_{2 y}$ are the elements of $P_{1}$ and $P_{2}$, respectively, i.e.,
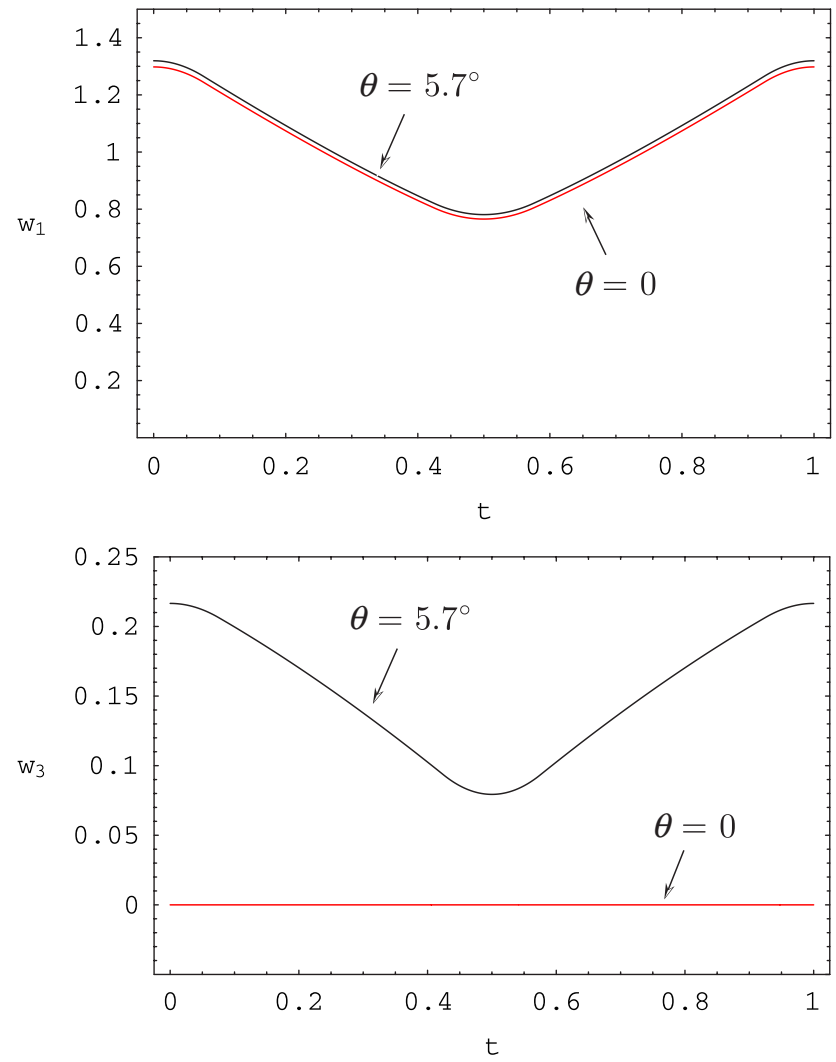

$$
P_{1}=\left(\begin{array}{cc}
P_{1 x} & P_{1 x y} \\
P_{1 y x} & P_{1 y}
\end{array}\right), \quad P_{2}=\left(\begin{array}{cc}
P_{2 x} & P_{2 x y} \\
P_{2 y x} & P_{2 y}
\end{array}\right)
$$

For the case without misalignment $(\theta=0)$, the matrices $P_{1}$ and $P_{2}$ are diagonal as expected, and the solutions are exactly those given by Eqs. (131)-(135). For $\theta=5.7^{\circ}, P_{1}$ and $P_{2}$ become nondiagonal, and their structures are more
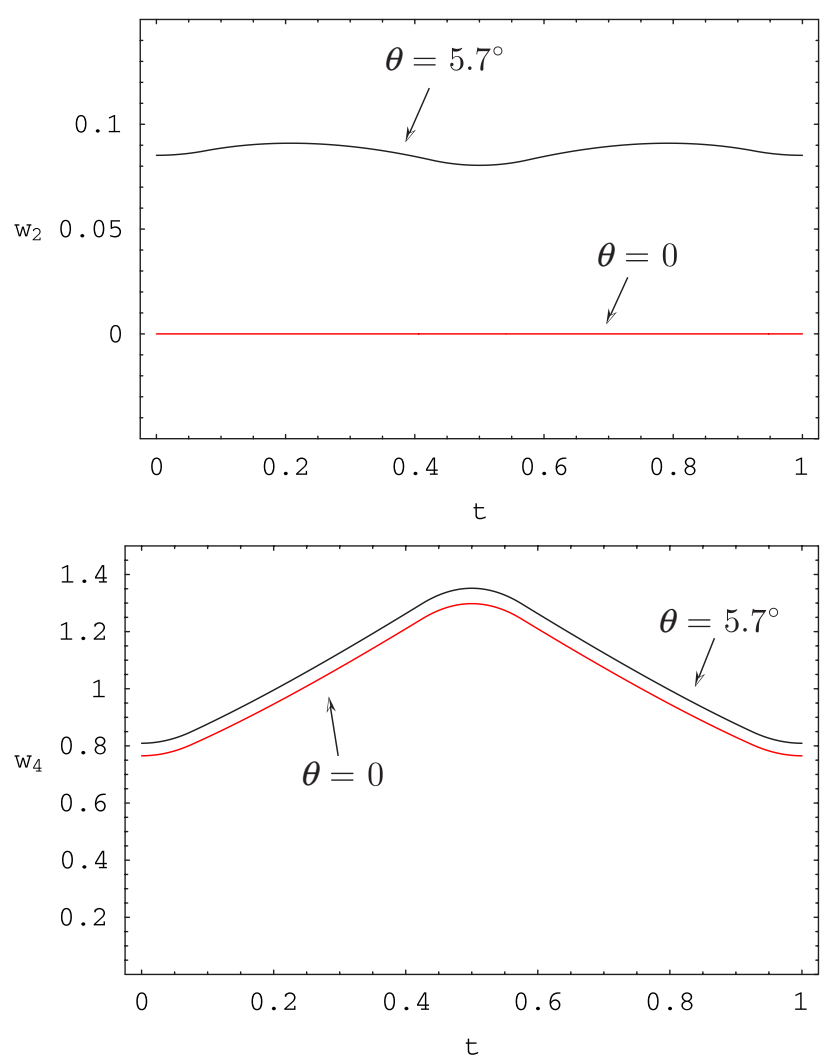

FIG. 2. (Color) The solutions for elements of the envelope matrix $w$ for the cases without misalignment $(\theta=0)$, and with a misalignment of $\theta=5.7^{\circ}$. The $t$ coordinate is normalized by the periodicity of the lattice. The $w_{1}$ and $w_{4}$ functions for $\theta=0$ are the familiar $x$ and $y$ envelopes for the uncoupled case. For $\theta=5.7^{\circ}$ the envelope matrix becomes nondiagonal due to the coupling between the two transverse degrees of freedom induced by the skew quadrupole component. 

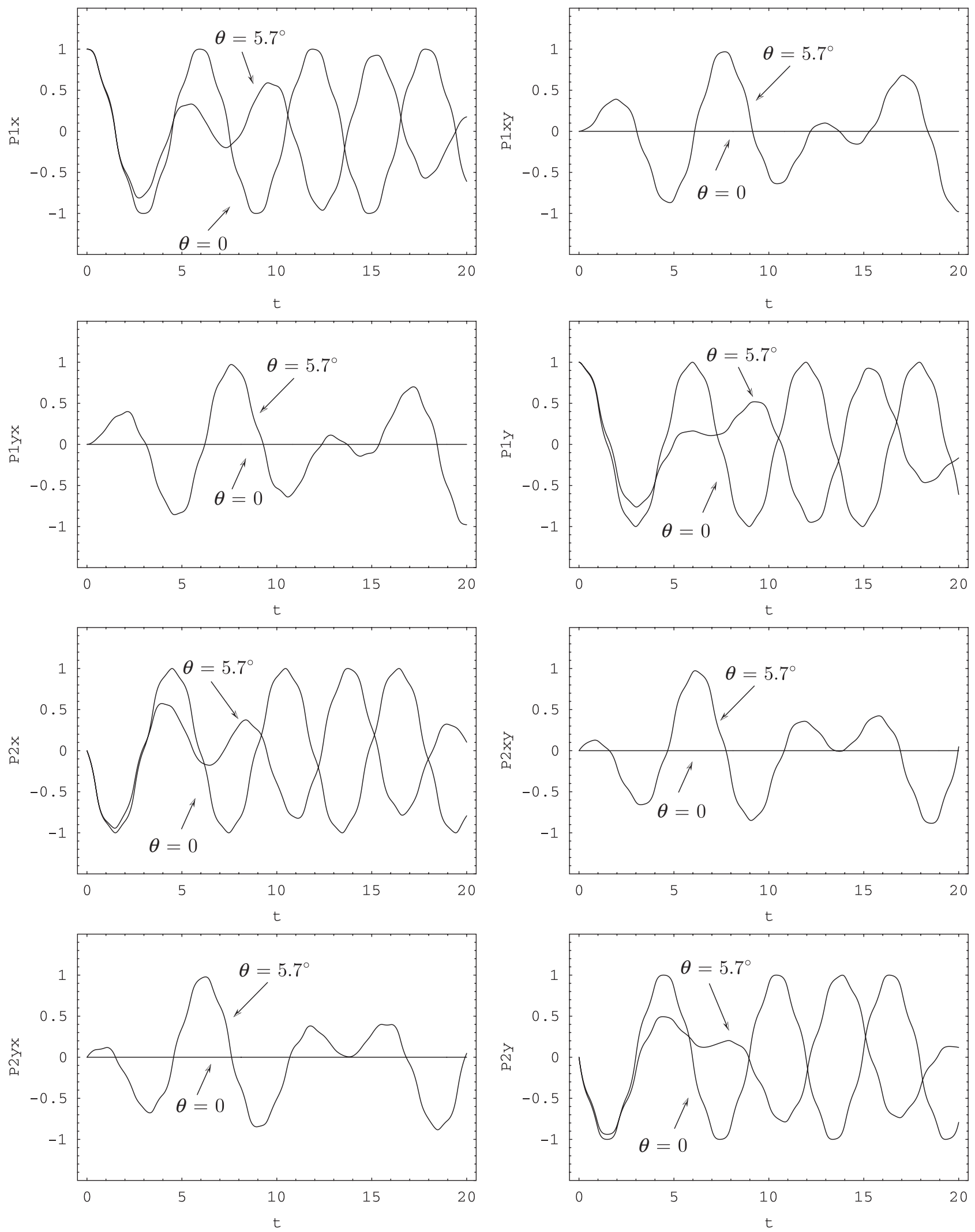

FIG. 3. The solutions for elements of the $P_{1}$ and $P_{2}$ matrices for the cases without misalignment $(\theta=0)$ and with a misalignment of $\theta=5.7^{\circ}$. The $t$ coordinate is normalized by the periodicity of the lattice. For the case without misalignment $(\theta=0)$, the matrices $P_{1}$ and $P_{2}$ are diagonal as expected, and the solutions are exactly those given by Eqs. (131)-(135). For $\theta=5.7^{\circ}, P_{1}$ and $P_{2}$ become nondiagonal, and their structures are more complex, as evident from the figure. 
complex. Finally, the transfer matrices are given by $w, P_{1}$, and $P_{2}$ according to Eq. (77).

As the second example of application of the generalized Courant-Snyder theory, we investigate the stability properties of the coupled dynamics. The most important issue of a strongly coupled system, such as a Möbius accelerator [40] or a final focusing system using solenoidal field $[37,38]$, is the stability of the coupled dynamics, an area where our theoretical understanding is very limited, owing to the lacking of a physics-based parametrization of the coupled dynamics. It is of course an elementary conclusion that the stability is completely determined by the symplectic transfer matrix $M$. Krein [41], Moser [42], and Yakubovich and Starzhinskii [43] studied in detail the stability properties of symplectic matrices. Using the theoretical formalism developed in this paper, we are able to deduce additional valuable information about the stability of the coupled dynamics. From Eq. (80), the one-turn map $M_{c T}(t)$ is similar to $P_{T}(t)$, and we conclude that the stability of the coupled dynamics can be determined by the phase advance matrix $P_{T}(t)$, without knowing the oneturn map $M_{c T}(t)$. This result simplifies the stability analysis, and underscores the physical importance of the phase advance $P_{T}(t)$. In addition, since $P_{T}(t)$ is a real $4 \mathrm{D}$ rotation, we obtain the following stability criterion: a necessary and sufficient condition for the coupled dynamics to be unstable is that the $P_{T}(t)$ matrix has an eigenvalue $\lambda$ with $|\lambda| \neq 1$. A second stability criterion for a strongly coupled system can be deduced by examining the invariant $I_{1}=$ $z^{T} U_{1} z=$ const, where

$$
U_{1}(t)=J M_{c T}-M_{c T}^{T} J=S^{T} P_{T}^{T} J\left[P_{T}^{T}-P_{T}\right] P_{T} S .
$$

If $U_{1}(t)$ is positive (negative) definite, then the amplitude of $z$ is bounded and the dynamics is stable. Equation (145) indicates that $U_{1}(t)$ and $J\left[P_{T}^{T}-P_{T}\right]$ are congruent. We thus obtain the following stability criterion determined from the antisymmetric component of the phase advance, i.e., $\left[P_{T}^{T}-P_{T}\right]$ : a sufficient condition for the coupled transverse dynamics to be stable is that $J\left[P_{T}^{T}-P_{T}\right]$ is positive or negative definite.

For the weakly coupled case, we show that the above criteria reduce to the known results of the sum and difference resonances. When the coupling effect is weak, it can be treated as a perturbation to the stable uncoupled dynamics. In order for the perturbed phase advance $P_{T}$ to have an eigenvalue $\lambda$ with $|\lambda| \neq 1$ for instability, the unperturbed phase advance $P_{T 0}$ must have two pairs of identical eigenvalues. Because

$$
\begin{gathered}
P_{T 0}=\left(\begin{array}{cc}
P_{1} & P_{2} \\
-P_{2} & P_{1}
\end{array}\right), \quad P_{1}=\left(\begin{array}{cc}
\cos \phi_{x} & 0 \\
0 & \cos \phi_{y}
\end{array}\right), \\
P_{2}=\left(\begin{array}{cc}
\sin \phi_{x} & 0 \\
0 & \sin \phi_{y}
\end{array}\right),
\end{gathered}
$$

this means that $\cos \phi_{x}=\cos \phi_{y}$, i.e., $\nu_{x} \pm \nu_{y}=n$, where $\nu_{x}$ and $\nu_{y}$ are tunes, and $n$ is an integer. This is the familiar sum and difference resonance condition. On the other hand, the sufficient condition for stability determined from $\left[P_{T}^{T}-P_{T}\right]$ in the weakly coupled case reveals that the difference resonance, or $\nu_{x}-\nu_{y}=n$, is stable. This is because a small perturbation due to weak coupling effect does not alter the positive (negative) definite character of $J\left[P_{T}^{T}-P_{T}\right]$. If the unperturbed $J\left[P_{T 0}^{T}-P_{T 0}\right]$ is positive (negative) definite, so is $J\left[P_{T}^{T}-P_{T}\right]$. We can easily see that when $\sin \phi_{x}=\sin \phi_{y}$, or $\nu_{x}-\nu_{y}=n, J\left[P_{T 0}^{T}-P_{T 0}\right]$ is positive definite. As a result, the difference resonance is stable. This leads us to the known result that only the sum resonance, i.e., $\nu_{x}+\nu_{y}=n$, can be unstable when there is a weak coupling effect. In this sense, the two stability criteria discovered by the theory developed here can be viewed as a generalization of the well-known results about sum and difference resonances for a weakly coupled system to a coupled system with arbitrary coupling strength.

\section{CONCLUSIONS}

Using a time-dependent canonical transformation technique, we have extended the Courant-Snyder theory for dynamics with one degree of freedom to the case of coupled transverse dynamics with 2 degrees of freedom. All of the quantities of physical importance in the original Courant-Snyder theory, including the envelope function, envelope equation, phase advance, transfer matrix, and the Courant-Snyder invariant, are elegantly generalized to the case of coupled transverse dynamics. The envelope function is generalized to a $2 \times 2$ envelope matrix, and the envelope equation is generalized to a matrix envelope equation. The envelope matrix determines a phase advance matrix, similar to the fact that, in the original CourantSnyder theory, the envelope function $w$ determines a phase advance function. The transfer matrix $M_{c}$ which solves the coupled transverse dynamics also has the same structure as the transfer matrix $M$ in the original Courant-Snyder theory, even though $M_{c}$ is a time-dependent $4 \mathrm{D}$ symplectic matrix, whereas $M$ is a time-dependent $2 \mathrm{D}$ symplectic matrix.

This generalized Courant-Snyder theory provides a new parametrization for the 4D symplectic transfer matrix $M_{c}$ [Eqs. (77) and (108)], which has a close connection to the original Courant-Snyder parametrization of the 2D symplectic matrix $M$ [Eq. (9)]. All of the parameters used in the generalized Courant-Snyder theory correspond to physical quantities of importance, and this parametrization can provide a valuable framework for accelerator design and particle simulation studies.

\section{ACKNOWLEDGMENTS}

This research was supported by the U.S. Department of Energy under Contract No. AC02-76CH03073. We thank 
Dr. Alex Dragt, Dr. Irving Haber, Dr. Moses Chung, Dr. John Barnard, Dr. Alex Chao, and Dr. Mei Bai for fruitful discussions.

[1] E. Courant and H. Snyder, Ann. Phys. (Paris) 3, 1 (1958).

[2] S. Y. Lee, Accelerator Physics (World Scientific, Singapore, 1999), p. 47.

[3] V. Ermakov, Univ. Izv. Kiev 20, 1 (1880).

[4] G. Birkhoff, Trans. Am. Math. Soc. 9, 219 (1908).

[5] W. E. Milne, Phys. Rev. 35, 863 (1930).

[6] E. Pinney, Proc. Am. Math. Soc. 1, 681 (1950).

[7] R. Kulsrud, Phys. Rev. 106, 205 (1957).

[8] H. Lewis, Jr., J. Math. Phys. (N.Y.) 9, 1976 (1968).

[9] K. Takayama, Phys. Lett. A 88, 57 (1982).

[10] H. Qin and R. C. Davidson, Phys. Rev. ST Accel. Beams 9 , 054001 (2006).

[11] H. Qin and R. C. Davidson, Phys. Rev. Lett. 96, 085003 (2006).

[12] D. A. Edwards and M. J. Syphers, An Introduction to the Physics of High-Energy Accelerators (Wiley, New York, 1993).

[13] J. J. Barnard, in Proceedings of the 1995 Particle Accelerators Conference (IEEE, Piscataway, NJ, 1996), p. 3241.

[14] Y. Cai, Phys. Rev. E 68, 036501 (2003).

[15] L. C. Teng, NAL Report No. FN-229, 1971.

[16] D. A. Edwards and L. C. Teng, IEEE Trans. Nucl. Sci. 20, 885 (1973).

[17] L.C. Teng, in Proceedings of the 2003 Particle Accelerator Conference (Piscataway, NJ, 2003), p. 2895.

[18] H. Grote, F. Iselin, E. Keil, and J. Niederer, in Proceedings of 1989 Particle Accelerator Conference (IEEE, Piscataway, NJ, 1989), p. 1292.

[19] G. Ripken, DESY Technical Report No. R1-70/04, 1970.

[20] H. Wiedemann, Particle Accelerator Physics II (SpringerVerlag, Berlin, 1995).

[21] D. Sagan and D. Rubin, Phys. Rev. ST Accel. Beams 2, 074001 (1999).

[22] A. Chao, in Proceedings of the 11th European Particle Accelerator Conference, Genoa, 2008 (EPS-AG, Genoa, Italy, 2008), p. 2963.

[23] H. Qin and R.C. Davidson, Phys. Plasmas 16, 050705
(2009).

[24] P. Leach, J. Math. Phys. (N.Y.) 18, 1608 (1977).

[25] G. Dattoli, C. Mari, M. Richetta, and A. Torre, Nuovo Cimento B 107, 269 (1992).

[26] G. Dattoli, G. Gallerano, C. Mari, A. Torre, and M. Richetta, Nuovo Cimento B 107, 1151 (1992).

[27] G. Dattoli, C. Mari, L. Mezi, and A. Torre, Nucl. Instrum. Methods Phys. Res., Sect. A 321, 447 (1992).

[28] A. J. Dragt, in Proceedings of the 1984 Summer Study of the Design and Utilization of the Superconducting Super Collider, edited by R. Donaldson and J. Morfin (Division of Particles and Fields of the American Physical Society, 1985).

[29] A. J. Dragt, F. Neri, G. Rangarajan, D. R. Douglas, L. M. Healy, and R. D. Ryne, Annu. Rev. Nucl. Part. Sci. 38, 455 (1988).

[30] A.J. Dragt, Handbook of Accelerator Physics and Engineering (World Scientific, Singapore, 1999), Chap. 2.3.6.

[31] A. J. Dragt (unpublished).

[32] A.J. Dragt, MARYLIE Manual (http://www.physics.umd. edu/dsat/) (2003).

[33] E. Forest, M. Berz, and J. Irwin, Part. Accel. 24, 91 (1989).

[34] M. Berz, in Proceedings of Nonlinear Effects in Accelerators, edited by M. Berz, S. Martin, and K. Ziegler (IOP Publishing, 1992), pp. 77-86.

[35] R. C. Davidson and H. Qin, Physics of Intense Charged Particle Beams in High Energy Accelerators (World Scientific, Singapore, 2001), p. 90.

[36] H. P. Manning, Geometry of Four Dimensions (MacMillan, London, 1914).

[37] B. G. Logan et al., Nucl. Instrum. Methods Phys. Res., Sect. A 577, 1 (2007).

[38] B. G. Logan et al., Nucl. Instrum. Methods Phys. Res., Sect. A (to be published).

[39] R. C. Davidson and H. Qin, Physics of Intense Charged Particle Beams in High Energy Accelerators (Ref. [35]), p. 58.

[40] R. Talman, Phys. Rev. Lett. 74, 1590 (1995).

[41] M. Krein, Dokl. Akad. Nauk. SSSR N.S. 73, 445 (1950).

[42] J. Moser, Commun. Pure Appl. Math. 11, 81 (1958).

[43] V. Yakubovich and V. Starzhinskii, Linear Differential Equations with Periodic Coefficients (Wiley, New York, 1975). 